\title{
Editorial
}

\section{The 1000th publication features protection of normal cells during chemotherapy: a land mark for Cell Death \& Disease}

\author{
Y Shi ${ }^{*, 1}$ \\ Cell Death and Disease (2014) 5, e1062; doi:10.1038/cddis.2014.50; published online 13 February 2014
}

As a sister of Cell Death \& Differentiation, Cell Death \& Disease was born in January 2010. In the last 4 years, through the great efforts of its editorial team, this new-born journal of the Nature Publication Group has received great support and trust from the scientific community around the world and its 1000th paper is now online. At this commemorative moment, we would like to express our sincere appreciations to the entire editorial team and all reviewers for their enthusiasm, dedication and determination in keeping high standards for the healthy growth of Cell Death \& Disease.

This peer-reviewed online journal is devoted to promote the field of emerging translational cell death research. The first 1000 publications focused on the integration of diverse areas of experimental and clinical medicine with specialties in cell death research related to cancer, immunity, stem cells and neuroscience. Its well-acknowledged cancer metabolism section and stem cell section have been an exceptional accomplishment of Cell Death \& Disease.

The translational spirit of the papers published in Cell Death \& Disease is exemplified by its 1000th publication. In this article, Becker et al. ${ }^{1}$ of the Li-Weber's research team at the German Cancer Research Center, Heidelberg, described their efforts in searching for compounds that could protect nonmalignant cells from DNA damage agent-induced cellular toxicity. This study is encouraged by a series of investigations using herbal extracts derived from transitional Chinese medicine (TCM) that could reduce the side effects of chemotherapy. Among various derivatives tested for the protection of human peripheral $\mathrm{T}$ cells from DNA damage agent-induced viability reduction, rocaglamide was found to be highly effective, significant protection could be observed at the nanomolar range.

Rocaglamide exists in the leaves of Aglaia sp. These plant species widely distributes in Southeast Asian countries and several of them have become ornamental plants. In TCM, Aglaia leaves have been used as anthelmintica, insecticide, and anti-inflammatory and anti-swelling medications. One of the derivative compounds that possess the anthelmintica and insecticidal activity is rocaglamide. Modern molecular and cellular biological investigations have revealed that this compound have strong anti-inflammatory activity through inhibiting NFKB in $T$ cells, anti-leukemic activity through blocking MAP kinase, induction of apoptosis through enhancing the expression of CD95L, and suppressing c-FLIP expression.

In the study by Becker et al., it was showed that rocaglamide significantly reduces apoptotic cell death induced by DNA-damaging anticancer drugs specifically in primary cells of mouse and human, including cardiomyocytes, $T$ cells, B cells and NK cells. It was found that rocaglamide specifically blocked DNA damage induced upregulation of p53 by inhibiting its protein synthesis. The role of such inhibition in rocaglamide-mediated protection was verified by knockdown of p53 and by comparison of the effects of rocaglamide on chemoprotection of splenocytes isolated from wild-type and p53-deficient mice. Importantly, this compound did not protect p53-deficient or -mutated cancer cells. Although the in vivo protective effect of rocaglamide needs to be further verified, this unique approach provides a novel mechanisms through which the side effects of chemotherapy could be reduced or

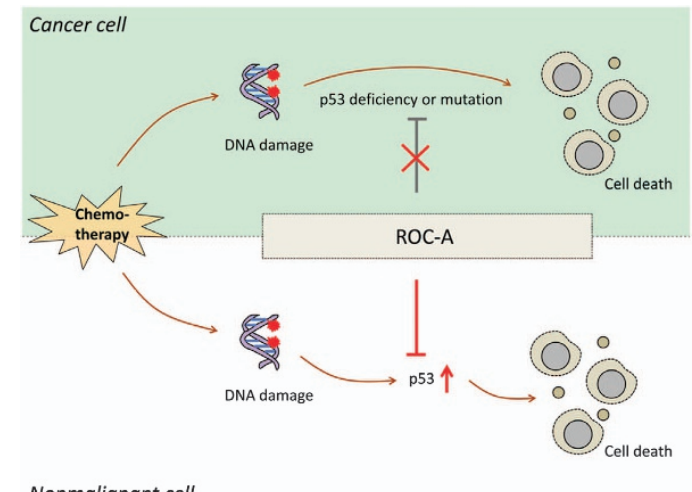

Figure 1 The chemoprotective ability of rocaglamide (ROC-A). It is demonstrated that the major mechanism of ROC-A protection against DNAdamage-induced cell death is dependent on its inhibition of p53 expression in nonmalignant cells. As such, in p53 deficiency or mutation cancer cells, ROC-A cannot exert such chemoprotective effects

\footnotetext{
${ }^{1}$ Key Laboratory of Stem Cell Biology, Institute of Health Sciences, Shanghai Institutes for Biological Sciences, Chinese Academy of Sciences \& Shanghai Jiao Tong University School of Medicine, Shanghai, China

*Corresponding author: Y Shi, Key Laboratory of Stem Cell Biology, Institute of Health Sciences, Shanghai Institutes for Biological Sciences, Chinese Academy of Sciences \& Shanghai Jiao Tong University School of Medicine, 225 South Chongqing Road, Shanghai 200025, China. Tel: +86 21 63848329; Fax: +86 21 63852655; E-mail: yufangshi@sibs.ac.cn
} 
eliminated. A diagrammatic summary of the study is presented in Figure 1.

According to the 2013 Thomson Reuters 2012 Journal Citation Reports Science Edition, Cell Death \& Disease achieved an impact factor of 6.044 and ranked number 35 among 184 journals in the cell biology field. Since its birth, Cell Death \& Disease has been striving to maintain its high standards, while keeping the pledged rapidity in publication through the online journalism. With an average of 20-25 papers published every month, the editorial team of Cell Death \& Disease has been carefully selecting the forefront cutting-research articles in the related fields. Clearly,
Cell Death \& Disease is well positioned to serve the translational biomedical community.

1. Becker MS, Schmezer P, Breuer R, Haas SF, Essers MA, Krammer PH et al. The traditional Chinese medical compound Rocaglamide protects nonmalignant primary cells from DNA damage-induced toxicity by inhibition of p53 expression. Cell Death Dis 2014; 5: e1000.

(c) (i) $(9)$ Cell Death and Disease is an open-access journal published by Nature Publishing Group. This work is licensed under a Creative Commons Attribution-NonCommercialNoDerivs 3.0 Unported License. To view a copy of this license, visit http://creativecommons.org/licenses/by-nc-nd/3.0/ 